\title{
The Third-Order Shear Deformation Theory for Modeling the Static Bending and Dynamic Responses of Piezoelectric Bidirectional Functionally Graded Plates
}

\author{
Nguyen Thai Dung, ${ }^{1}$ Phung Van Minh $\mathbb{D}^{2},{ }^{2}$ Hoang Manh Hung, ${ }^{2}$ and Dao Minh Tien ${ }^{3}$ \\ ${ }^{1}$ Department of Special Equipment, Faculty of Special Equipment, Le Quy Don Technical University, Hanoi, Vietnam \\ ${ }^{2}$ Faculty of Mechanical Engineering, Le Quy Don Technical University, Hanoi, Vietnam \\ ${ }^{3}$ Airforce-Air Defence Technical Institude, Hanoi, Vietnam
}

Correspondence should be addressed to Phung Van Minh; minhpv.lqdtu@gmail.com

Received 2 February 2021; Revised 1 May 2021; Accepted 29 May 2021; Published 8 June 2021

Academic Editor: Hongtao Zhu

Copyright (c) 2021 Nguyen Thai Dung et al. This is an open access article distributed under the Creative Commons Attribution License, which permits unrestricted use, distribution, and reproduction in any medium, provided the original work is properly cited.

\begin{abstract}
This work is the first exploration of the static bending and dynamic response analyses of piezoelectric bidirectional functionally graded plates by combining the third-order shear deformation theory of Reddy and the finite element approach, which can numerically model mechanical relations of the structure. The present approach and mechanical model are confirmed through the verification examples. The geometrical and material study is conducted to evaluate the effects of the feedback coefficients, volume fraction parameter, and constraint conditions on the static and dynamic behaviors of piezoelectric bidirectional functionally graded structures, and this work presents a wide variety of static and dynamic behaviors of the plate with many interesting results. There are many meanings that have not been mentioned by any work, especially the working performance of the structure is better than that when the feedback parameter of the piezoelectric component is added, that is, the piezoelectric layer increases the working efficiency. Numerical investigations are the important basis for calculating and designing related materials and structures in technical practice.
\end{abstract}

\section{Introduction}

In recent decades, due to the science and technology are developed so rapidly, many intelligent materials appear to serve the complicated requirements in engineering practice such as functionally graded materials (FGMs), piezoelectrics, electroactive polymers, magnetostrictive, and shape memory alloys, in which functionally graded materials are one of the most important and popular structures, which are used and considered widely by scientists worldwide. Traditionally, FGMs are a remarkable type of composite structure, where the material characteristics change gradually and smoothly from one surface to the other ones [1-4], where they can be dealt with and designed for special duties and applications. A huge number of methods, which are founded by the particulate process, layer process, and melting process are employed to manufacture the FG structures [5]. In recent years, scientists have used two-dimensional functionally graded materials to significantly lessen the thermal stress phenomena in machine parts that are subjected to intent thermal loads [6-9]. Moreover, as mentioned above, hightech structures require complex technical conditions; therefore, one structure may need to integrate many kinds of smart materials, for example, piezoelectric bidirectional functionally graded structures [10-12]. This is also the mixed material plate investigated in this study.

Before focusing on the main purpose of this paper, some reviews of piezoelectric bidirectional functionally graded structures and related ones are discussed. For the analysis of the static bending phenomena, Thom et al. [10] introduced an analysis of two-directional FG plates by using the FE method (FEM) and a developed third-order shear theory 
(TSDT). In their investigation, the plate kinematics were expressed by using the TSDT without any shear correction coefficients. The third-order shear deformation theory also relied on the scrupulous kinematic of displacement components, which was presented to govern over other aforementioned approaches and established based on elastic formulations. The material characteristics were simulated to be changed smoothly in two directions, and their effective characteristics were calculated through the law of commixture. Tam et al. [11] firstly presented the material optimization of the acclimatization minimization of twodirectional FG structures subjected to static loads. In this approach, the distribution of the two-directional FG mechanical characteristics was represented by using the $2 \mathrm{D}$ nonuniform rational B-spline (NURBS) functions. In addition, 1-D NURBS fundamental functions were employed to present the accurate structural parameter and presume the unrevealed solution in the FE analysis (FEA), where design parameters were the ceramic capacity fraction coefficients at driving points by the $x$ and $z$ directions established by the isogeometric analysis (IGA) approach. The optimization issue was solved by a tool called the differential evolution (DE) algorithm. For vibration analyses, Jinqiang et al. [12] considered active oscillation control of the piezoelectric FG plate by employing the piezoelectricity material as an actuator, in which the Kirchhoff multilayered plate theory was employed, and the equation of motion for the piezoelectric FG plate was derived by using Rayleigh-Ritz approach and Hamilton's principle. Wankhade and Bajoria [13] studied the vibration behaviors of piezoelectric laminated composite plates with the coupled electromechanical actuation, in which the efficient FE formulation with the higher-order shear deformation (HSDT) plate theory was used to combine the influences of shear strain components in the computational model. An isoperimetric 8-noded rectangular plate element was employed based on the linear electric potential distribution by the thickness direction to derive the mechanical relations. Nam et al. [14] investigated free and forced oscillation behaviors of clever functionally graded plates, which were stiffened by adding graphene platelets (GPLs). Then, the active control of FG plates with piezoelectricity layers was analyzed. To contribute numeral solutions to fundamental issues, a computational strategy with a $C^{0}$-HSDT polygonal FE formulation (PFEM) was developed, which was suitable for simulating both thin and thick structures. And, for dynamic problems, some important works can be counted as follows. Yas et al. [15] used a semianalytical method called the state space-based differential quadrature method (SSDQM) to investigate the 3D free oscillation of a multidirectional FG piezoelectric (FGP) annular plate structure, which was rested on Pasternak's two-parameter elastic foundations. Wu et al. [16] introduced a review of 3D analytical approaches for the investigation of multilayered and FG piezoelectric plate and shell structures. The literature considered the 3D analysis of multilayered and FG piezoelectric plates with 191 references. Yongbo et al. [17] studied analytically the deflection field of piezoelectric 2D-FGM structures, where the material characteristics varied exponentially both along with the longitudinal and horizontal directions. The double equations were introduced by variation rule and presenting segmentation of variables was employed. Then, in the complex space, which was consisted of the initial and dual variables, the problem could be solved by using the symplectic development. Rasool et al. [18] studied the abridged dynamic displacements of a developed piezoelectric sandwich structure subjected to the mix of thermo-electro-mechanical loads. In the considered piezoelectric sandwich structure, two active piezoelectric components were added to the surfaces of a submissive advanced plate with two thin GRNC layers and a thick porous composite core. In the GRNC sheets, the graphene component was considered to be changed by the functional dismemberment rules. By employing developed Halpin-Tsai equations, the thermal-dependent mechanical characteristics of nanocomposite layers have been defined. Reddy's third-order shear deformation plate theory (called TSDT) was used to establish the dual reigning equations, and MLS shape functions were employed in the meshless method. Wang and Huang [19] investigated analytically the dynamic responses of piezoelectric sensor components placed in an elastic foundation subjected to the electromechanical loads persuaded by piezoelectricity layers. A sensor/actuator mechanical model considering the deformation in two longitudinal and transverse directions of the piezoelectric layers was modified. The dynamic loading transfer between the piezoelectricity layers and the elastic medium was examined by using a Fourier transform approach and integral expressions in terms of the interfacial shear and normal stress components. Quang and his co-workers [20] examined the static and dynamic response of thin and moderate thickness plates based on a cell-based smoothed discrete shear gap method (CS-DSG3) using the FSDT. In which, the CS-DSG3 wasdeveloped for investigation and active oscillation control of the functionally graded plates with piezoelectric sensor and actuator components. Besides, mechanical behaviors of the rod, plate, and shell structures taking into account the piezoelectricity were presented abundantly in documents [25-27].

To deal with the structures made of functionally graded materials resting on elastic foundations, most recently, some works used the HSDT to simulate the static bending, the buckling, and free vibration of these structures, readers can find relevant studies in [21-23].

To the best of our best knowledge, there seems to be no papers dealing with the static bending and dynamic behavior of piezoelectric two-directional FG material (2D-FGM) plates; however, this is an interesting issue with a great help in applying this structure in engineering practice, which can be used to make aircraft wings, turbines, etc. Therefore, in this paper, both the static and dynamic problems of this mixed structure are carried out by using the FEM based on the TSDT of Reddy. This is a computational theory that does not need any shear correction coefficients but still ensures the accuracy of the simulation. This paper also shows that when the feedback coefficients of the piezoelectric effect are added, the performance of the plate increases significantly, in the other words, the vibration reduction is better, and 
therefore, increasing the efficiency of use against structures with variable mechanical properties are integrated with piezoelectricity layers.

The rest of this work is structured into the following four sections. Section 2 briefly introduces piezoelectric bidirectional FG plates. Section 3 presents a clear FE formulation for piezoelectricity 2D-FGM plate structures. Section 4 shows the accuracy problems and numerical explorations of both static bending and dynamic response analyses of the plate. Some new explorations are summed up in Section 5.

\section{Piezoelectric Bidirectional Functionally Graded Plates}

This paper focuses on the static and dynamic behaviors of the piezoelectric $2 \mathrm{D}$-FGM plate with the core layer made from three different materials, which are represented as 1,2 , and 3, respectively. Material characteristics including Poisson's ratio, Young's modulus, and mass density are $v_{i}, E_{i}$, and $\rho_{i}(i=1-3)$. Two outer surfaces are the piezoelectricity actuator and sensor layers in Figure 1.

The mechanical characteristics of the core layer are contingent on the volume fraction exponents of three different materials as follows [10]:

$$
\left\{\begin{array}{l}
E(x, z)=E_{1} V_{1}+E_{2} V_{2}+E_{3} V_{3}, \\
\nu(x, z)=v_{1} V_{1}+v_{2} V_{2}+\nu_{3} V_{3}, \\
\rho(x, z)=\rho_{1} V_{1}+\rho_{2} V_{2}+\rho_{3} V,
\end{array}\right.
$$

where $V_{i}(i=1-3)$ are the volume fraction of materials, and [10]

$$
\left\{\begin{array}{l}
V_{1}=\left[1-\left(\frac{z}{h}+\frac{1}{2}\right)^{n}\right]\left[1-\left(\frac{x}{a}\right)^{q}\right] \\
V_{2}=\left[1-\left(\frac{z}{h}+\frac{1}{2}\right)^{n}\right]\left(\frac{x}{a}\right)^{q} \\
\mathrm{~V}_{3}=\left(\frac{z}{h}+\frac{1}{2}\right)^{n}
\end{array}\right.
$$

in which, $n$ and $q \geq 0$ are the volume fraction indices.

\section{Finite Element Formulation of Piezoelectricity 2D-FGM Plates}

Herein, the TSDT of Reddy is employed; thus, the displacement components at one point in the structure are expressed as [24]

$$
\left\{\begin{array}{l}
u(x, y, z)=u_{0}(x, y)+z j_{x}(x, y)-\frac{4}{3 h^{2}} z^{3}\left(j_{x}+\frac{\partial w_{0}}{\partial x}\right) \\
v(x, y, z)=v_{0}(x, y)+z j_{y}(x, y)-\frac{4}{3 h^{2}} z^{3}\left(j_{y}+\frac{\partial w_{0}}{\partial y}\right) \\
w(x, y, z)=w_{0}(x, y)
\end{array}\right.
$$

with $\mathbf{u}_{0}, \mathbf{v}_{0}$, and $\mathbf{w}_{0}$ are the displacement components at $(x$, $y, z=0)$ and $\varphi_{x}$ an $d \varphi_{y}$ are the transversal normal rotations in $x z$ and $y z$ surfaces.

The strain parts are calculated as

$$
\left\{\begin{array}{l}
\varepsilon_{x}=\varepsilon_{x}^{m}+z \kappa_{x}^{1}+z^{3} \kappa_{x}^{3}, \\
\varepsilon_{y}=\varepsilon_{y}^{m}+z \kappa_{y}^{1}+z^{3} \kappa_{y}^{3}, \\
\gamma_{x y}=\gamma_{x y}^{0}+z \kappa_{x y}^{1}+z^{3} \kappa_{x y}^{3}, \\
\gamma_{x z}=\gamma_{x z}^{0}+z^{2} \gamma_{x z}^{2}, \\
\gamma_{y z}=\gamma_{y z}^{0}+z^{2} \gamma_{y z}^{2} .
\end{array}\right.
$$

Equation (4) is then shortened in the matrix expression as

$$
\begin{aligned}
\varepsilon^{m} & =\left\{\begin{array}{c}
\varepsilon_{x}^{m} \\
\varepsilon_{y}^{m} \\
\gamma_{x y}^{m}
\end{array}\right\} \\
& =\left\{\begin{array}{c}
\frac{\partial u_{0}}{\partial x} \\
\frac{\partial v_{0}}{\partial y} \\
\frac{\partial v_{0}}{\partial x}+\frac{\partial u_{0}}{\partial y}
\end{array}\right\},
\end{aligned}
$$




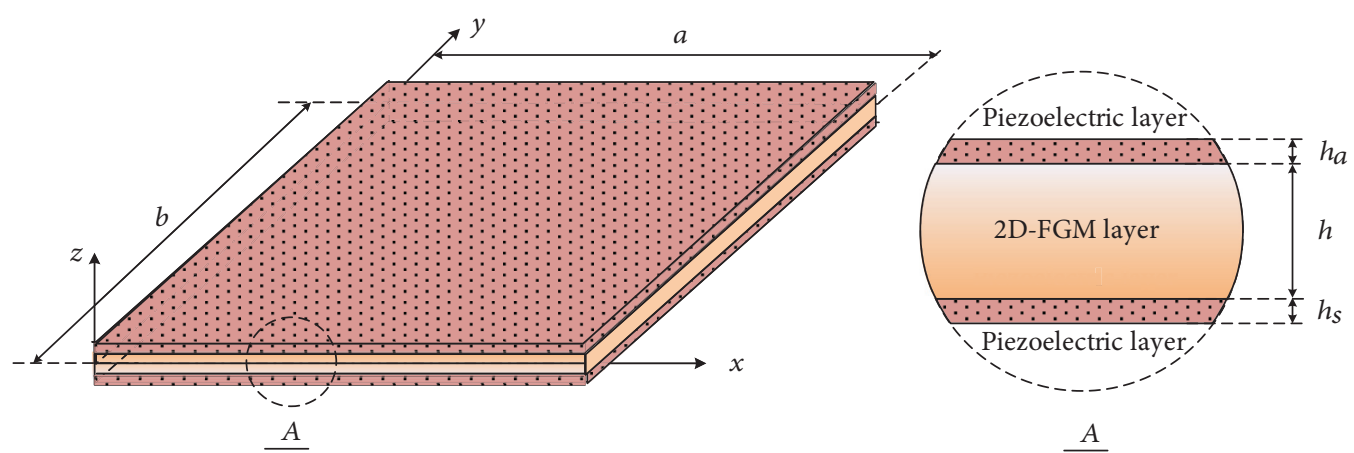

Figure 1: The general representation of the piezoelectric 2D-FGM plate and its $x \mathrm{O} z$ cross section.

$$
\begin{aligned}
\kappa_{x}^{1}=\left\{\begin{array}{c}
\kappa_{x}^{1} \\
\kappa_{y}^{1} \\
\kappa_{x y}^{1}
\end{array}\right\}=\left\{\begin{array}{c}
\frac{\partial \varphi_{x}}{\partial x} \\
\frac{\partial \varphi_{y}}{\partial y} \\
\frac{\partial \varphi_{x}}{\partial y}+\frac{\partial \varphi_{y}}{\partial x}
\end{array}\right\}, \kappa_{x}^{3}=\left\{\begin{array}{c}
\kappa_{x}^{3} \\
\kappa_{y}^{3} \\
\kappa_{x y}^{3}
\end{array}\right\} \\
=\frac{-4}{3 h^{2}}\left\{\begin{array}{c}
\frac{\partial \varphi_{x}}{\partial x}+\frac{\partial^{2} w_{0}}{\partial x^{2}} \\
\frac{\partial \varphi_{y}}{\partial y}+\frac{\partial^{2} w_{0}}{\partial y^{2}} \\
\frac{\partial \varphi_{x}}{\partial y}+\frac{\partial \varphi_{y}}{\partial x}+\frac{2 \partial^{2} w_{0}}{\partial x \partial y}
\end{array}\right\}, \gamma^{0}=\left\{\begin{array}{c}
\gamma_{x z}^{0} \\
\gamma_{y z}^{0}
\end{array}\right\} \\
=\left\{\begin{array}{c}
\varphi_{x}+\frac{\partial w_{0}}{\partial x} \\
\varphi_{y}+\frac{\partial w_{0}}{\partial y}
\end{array}\right\}, \gamma^{2}=\left\{\begin{array}{c}
\gamma_{x z}^{2} \\
\gamma_{y z}^{2}
\end{array}\right\}=\frac{-4}{h^{2}}\left\{\begin{array}{c}
\varphi_{x}+\frac{\partial w_{0}}{\partial x} \\
\varphi_{y}+\frac{\partial w_{0}}{\partial y}
\end{array}\right\} .
\end{aligned}
$$

The linear piezoelectric fastening between the electric field and the elastic field can be defined by the converse piezoelectric and direct equations and the constitutive and electric displacement relations [21, 22, 24-30].

For the core 2D-FGM layer,

$$
\left\{\begin{array}{l}
\sigma=D_{b}\left(\varepsilon^{m}+z \kappa^{1}+z^{3} \kappa^{3}\right), \\
\tau=D_{s}\left(\gamma^{0}+z^{2} \gamma^{2}\right) .
\end{array}\right.
$$

For the two piezoelectric layers,

$$
\left\{\begin{array}{l}
\sigma=D_{b p}\left(\varepsilon^{m}+z \kappa^{1}+z^{3} \kappa^{3}\right)-e^{T} E_{v}, \\
\tau=D_{s p}\left(\gamma^{0}+z^{2} \gamma^{2}\right), \\
D=e\left(\varepsilon^{0}+z \varepsilon^{1}+z^{3} \varepsilon^{3}\right)+p E_{v},
\end{array}\right.
$$

where

$$
\begin{gathered}
\sigma=\left\{\begin{array}{c}
\sigma_{x} \\
\sigma_{y} \\
\tau_{x y}
\end{array}\right\}, \\
\tau=\left\{\begin{array}{c}
\tau_{x z} \\
\tau_{y z}
\end{array}\right\} .
\end{gathered}
$$

For the core 2D-FGM layer,

$$
\begin{aligned}
& D_{b}=\frac{E(x, z)}{1-v^{2}(x, z)}\left[\begin{array}{ccc}
1 & v(x, z) & 0 \\
v(x, z) & 1 & 0 \\
0 & 0 & \frac{1-\nu(x, z)}{2}
\end{array}\right], \\
& D_{s}=\frac{E(x, z)}{2(1+v(x, z))}\left[\begin{array}{ll}
1 & 0 \\
0 & 1
\end{array}\right] .
\end{aligned}
$$

For the two surface piezoelectric layers,

$$
\begin{aligned}
& D_{b p}=\frac{E_{p}}{1-v_{p}^{2}}\left[\begin{array}{ccc}
1 & v_{p} & 0 \\
v_{p} & 1 & 0 \\
0 & 0 & \frac{1-v_{p}}{2}
\end{array}\right], \\
& D_{s p}=\frac{E_{p}}{2\left(1+v_{p}\right)}\left[\begin{array}{ll}
1 & 0 \\
0 & 1
\end{array}\right] .
\end{aligned}
$$

So, the stiffness coefficients $D_{b}$ and $D_{s}$ vary in both $x$ - and $y$-directions, and this is completely different from the $1 \mathrm{D}$ FGM plate.

$-E_{\mathrm{v}}$ is the component of the electricity, where it is calculated by the transformation of the potential as [23] 


$$
\begin{aligned}
E_{\mathrm{v}} & =-\nabla \phi \\
& =\left\{\begin{array}{c}
0 \\
0 \\
E^{z}
\end{array}\right\} .
\end{aligned}
$$

In this work, it is considered that the electric field is applied only to the structure in the depth direction, and the symbol $E_{z}$ is the electric component, which is calculated as

$$
E^{z}=\frac{-\phi}{h_{p}} .
$$

$-D$ is the displacement vector due to the electricity; $-e$ represents the stress coefficients related to the piezoelectricity, which has the following specific expression:

$$
e=\left[\begin{array}{ccc}
0 & 0 & 0 \\
0 & 0 & 0 \\
e_{31} & e_{32} & 0
\end{array}\right] .
$$

$-p$ is the component (tensor) of the dielectricity at unchanged mechanistic strain, which has the description as follows:

$$
p=\left[\begin{array}{ccc}
p_{11} & 0 & 0 \\
0 & p_{22} & 0 \\
0 & 0 & p_{33}
\end{array}\right]
$$

The internal load can be presented as

$$
\left\{\begin{array}{c}
N \\
M \\
\bar{M} \\
Q \\
\bar{Q}
\end{array}\right\}=\left[\begin{array}{ccccc}
A & B & E & 0 & 0 \\
B & D & F & 0 & 0 \\
E & F & H & 0 & 0 \\
0 & 0 & 0 & \bar{A} & \bar{B} \\
0 & 0 & 0 & \bar{B} & \bar{D}
\end{array}\right]\left\{\begin{array}{c}
\varepsilon^{m} \\
\kappa^{1} \\
\kappa^{3} \\
\gamma^{0} \\
\gamma^{2}
\end{array}\right\},
$$

in which

$$
\begin{aligned}
& \left\{\begin{array}{l}
A \\
B \\
D \\
E \\
F \\
H
\end{array}\right\}=\int_{-h / 2}^{h / 2} D_{b}\left\{\begin{array}{c}
1 \\
z \\
z^{2} \\
z^{3} \\
z^{4} \\
z^{6}
\end{array}\right\} \mathrm{d} z+\int_{-h_{s}-h / 2}^{-h / 2} D_{b p}\left\{\begin{array}{c}
z^{2} \\
z^{3} \\
z^{4} \\
z^{6}
\end{array}\right\} \mathrm{d} z+\int_{h / 2}^{h / 2+h_{a}} D_{b p}\left\{\begin{array}{c}
1 \\
z \\
z^{2} \\
z^{3} \\
z^{4} \\
z^{6}
\end{array}\right\} \mathrm{d} z, \\
& \left\{\begin{array}{l}
\bar{A} \\
\bar{B} \\
\bar{D}
\end{array}\right\}=\int_{-h / 2}^{h / 2} D_{s}\left\{\begin{array}{c}
1 \\
z^{2} \\
z^{4}
\end{array}\right\} \mathrm{d} z+\int_{-h_{s}-h / 2}^{-h / 2} D_{s p}\left\{\begin{array}{c}
1 \\
z^{2} \\
z^{4}
\end{array}\right\} \mathrm{d} z \int_{h / 2}^{h / 2+h_{a}} D_{s p}\left\{\begin{array}{c}
1 \\
z^{2} \\
z^{4}
\end{array}\right\} \mathrm{d} z .
\end{aligned}
$$

This work employs the plate element of four-node, and each one contains five DOFs:

$$
\begin{aligned}
& u_{0}=\sum_{j=1}^{4} N_{j}(\xi, \eta) \cdot u_{j}, \\
& v_{0}=\sum_{j=1}^{4} N_{j}(\xi, \eta) \cdot v_{j}, \\
& w_{0}=\sum_{j=1}^{4} N_{j}(\xi, \eta) \cdot w_{0 j}, \\
& \varphi_{x}=\sum_{j=1}^{4} N_{j}(\xi, \eta) \cdot \varphi_{x j}, \\
& \varphi_{y}=\sum_{j=1}^{4} N_{j}(\xi, \eta) \cdot \varphi_{y j},
\end{aligned}
$$


where $N_{j}$ is the interpolation functions (Lagrangian type) for the four-node plate element and $\xi$ and $\eta$ are the values in the natural coordinate system.

According to the previous interpolation method, the strain fields are shortened as

$$
\left\{\begin{array}{l}
\varepsilon^{m}=B_{0} q_{e} ; \kappa^{1}=B_{1} q_{e} ; \kappa^{3}=B_{3} q_{e}, \\
\gamma^{0}=B_{0 \gamma} q_{e} ; \gamma^{2}=B_{2 \gamma} q_{e},
\end{array}\right.
$$

with

$$
B_{0}=\sum_{j=1}^{4}\left[\begin{array}{ccccc}
\frac{\partial N_{j}}{\partial x} & 0 & 0 & 0 & 0 \\
0 & \frac{\partial N_{j}}{\partial y} & 0 & 0 & 0 \\
\frac{\partial N_{j}}{\partial y} & \frac{\partial N_{j}}{\partial x} & 0 & 0 & 0
\end{array}\right],
$$$$
B_{1}=\sum_{j=1}^{4}\left[\begin{array}{ccccc}
0 & 0 & 0 & \frac{\partial N_{j}}{\partial x} & 0 \\
0 & 0 & 0 & 0 & \frac{\partial N_{j}}{\partial y} \\
0 & 0 & 0 & \frac{\partial N_{j}}{\partial y} & \frac{\partial N_{j}}{\partial x}
\end{array}\right],
$$$$
B_{3}=\frac{-4}{3 h^{2}} \sum_{j=1}^{4}\left[\begin{array}{ccccc}
0 & 0 & \frac{\partial^{2} N_{j}}{\partial x^{2}} & \frac{\partial N_{j}}{\partial x} & 0 \\
0 & 0 & \frac{\partial^{2} N_{j}}{\partial y^{2}} & 0 & \frac{\partial N_{j}}{\partial y} \\
0 & 0 & 2 \frac{\partial^{2} N_{j}}{\partial x \partial y} & \frac{\partial N_{j}}{\partial y} & \frac{\partial N_{j}}{\partial x}
\end{array}\right],
$$

$$
\begin{gathered}
B_{0 \gamma}=\sum_{j=1}^{4}\left[\begin{array}{lllll}
0 & 0 & \frac{\partial N_{j}}{\partial x} & 0 & N_{j} \\
0 & 0 & \frac{\partial N_{j}}{\partial y} & N_{j} & 0
\end{array}\right], \\
B_{2 \gamma}=\frac{-4}{h^{2}} \sum_{j=1}^{4}\left[\begin{array}{lllll}
0 & 0 & \frac{\partial N_{j}}{\partial x} & 0 & N_{j} \\
0 & 0 & \frac{\partial N_{j}}{\partial y} & N_{j} & 0
\end{array}\right] .
\end{gathered}
$$


Then, the electric field vectors (in the sensor and actuator layers) are defined by two degrees of freedom $\phi_{a}$ and $\phi_{s}$ as

$$
\begin{aligned}
E_{v} & =-\left[\begin{array}{c}
0,0, \frac{1}{h_{a}} \\
0,0, \frac{1}{h_{s}}
\end{array}\right]^{T}\left\{\begin{array}{l}
\phi_{a} \\
\phi_{s}
\end{array}\right\} \\
& =-\left[\begin{array}{c}
B_{\phi}^{a} \\
B_{\phi}^{s}
\end{array}\right]\left\{\begin{array}{c}
\phi_{a} \\
\phi_{s}
\end{array}\right\},
\end{aligned}
$$

where

$$
\begin{aligned}
B_{\phi}^{a} & =\left[0,0, \frac{1}{h_{a}}\right]^{T}, \\
B_{\phi}^{s} & =\left[0,0, \frac{1}{h_{s}}\right]^{T},
\end{aligned}
$$

where $h_{s}$ and $h_{a}$ are the depths of the sensor and actuator layers.

The whole strain energy of the piezoelectric FG plate can be computed as

$$
\begin{aligned}
& \Pi_{e}= \frac{1}{2} \int_{V_{e}}\left(\left\{\varepsilon^{0} \varepsilon^{1} \varepsilon^{3}\right\}^{T}+\left\{\gamma^{0} \gamma^{2}\right\}^{T} \tau-D^{T} E_{v}\right) \mathrm{d} V-\int_{S_{e}} u^{T} \widehat{F} \mathrm{~d} S-\int_{V_{e}} E_{v}^{T} \overline{\mathrm{Q}} \mathrm{d} V= \\
&= q_{e}^{T} \frac{1}{2} \int_{S_{e}}\left(\begin{array}{c}
B_{0}^{T} A B_{0}+B_{0}^{T} B B_{1}+B_{1}^{T} E B_{3}+B_{1}^{T} B B_{0}+B_{1}^{T} D B_{1}+B_{0}^{T} E B_{3} \\
+B_{3}^{T} E B_{0}+B_{1}^{T} F B_{3}+B_{3}^{T} E B_{1}+B_{3}^{T} F B_{1}+B_{3}^{T} H B_{3} \quad \bar{A} B_{0 \gamma}+B_{0 \gamma}^{T} \bar{B} B_{2 \gamma}+B_{2 \gamma}^{T} \bar{B} B_{0 \gamma}+B_{2 \gamma}^{T} \bar{B} B_{2 \gamma}
\end{array}\right) \mathrm{d} q_{e} \\
&-q_{e}^{T} \frac{1}{2} \int_{S_{e}}\left(-B_{0}^{T} A e^{T} B_{\phi a}-B_{1}^{T} B e^{T} B_{\phi a}-B_{3}^{T} E e^{T} B_{\phi a}\right) \mathrm{d} S \phi_{e a} \\
&-q_{e}^{T} \frac{1}{2} \int_{S_{e}}\left(-B_{0}^{T} A e^{T} B_{\phi s}-B_{1}^{T} B e^{T} B_{\phi s}-B_{3}^{T} E e^{T} B_{\phi s}\right) \mathrm{d} S \phi_{e s} \\
&-\phi_{e a}^{T} \frac{1}{2} \int_{S_{e}}\left(-B_{\phi a}^{T} e A B_{0}-B_{\phi a}^{T} e B B_{1}-B_{\phi a}^{T} e E B_{3}\right) \mathrm{d} S q_{e} \\
&-\phi_{e s}^{T} \frac{1}{2} \int_{S_{e}}\left(-B_{\phi s}^{T} e A B_{0}-B_{\phi s}^{T} e B B_{1}-B_{\phi s}^{T} e E B_{3}\right) \mathrm{d} S q_{e} \\
&-\frac{1}{2} \phi_{e a}^{T} \int_{S_{e}} B_{\phi a}^{T} p B_{\phi a}^{T} \mathrm{~d} S \phi_{e}^{a}-\frac{1}{2} \phi_{e s}^{T} \int_{S_{e}} B_{\phi s}^{T} p B_{\phi s}^{T} \mathrm{~d} S \phi_{e s} \\
&-q_{e}^{T} \int_{S_{e}} N^{T} \widehat{F} \mathrm{~d} S-\int_{V_{e}} E_{v}^{T} \bar{Q} \mathrm{~d} V, \\
&
\end{aligned}
$$

where $\widehat{F}$ is the facade load, and expression (22) is presented in matrix configuration as

$$
\prod_{e}=\frac{1}{2}\left(\begin{array}{c}
q_{e}^{T} K_{u u e} q_{e}+q_{e}^{T} K_{u \phi e}^{a} \phi_{a} e+q_{e}^{T} K_{u \phi e}^{s} \phi_{s e} \\
+\phi_{a e}^{T} K_{\phi u e}^{a} q_{e}+\phi_{s} e_{T} K_{\phi u e}^{s} q_{e} \\
-\phi_{a e}^{T} K_{\phi \phi e}^{a} \phi_{a e}-\phi_{s e}^{T} K_{\phi \phi e}^{s} \phi_{s e}
\end{array}\right)-q_{e}^{T} P_{e}-\phi_{a e}^{T} F_{q e}^{a}-\phi_{s e}^{T} F_{q e}^{s},
$$


where the stiffness matrices are defined as

$$
\begin{aligned}
K_{\text {uиe }} & =\int_{S_{e}}\left(\begin{array}{c}
B_{0}^{T} A B_{0}+B_{0}^{T} B B_{1}+B_{1}^{T} E B_{3}+B_{1}^{T} B B_{0}+B_{1}^{T} D B_{1}+B_{0}^{T} E B_{3} \\
+B_{3}^{T} E B_{0}+B_{1}^{T} F B_{3}+B_{3}^{T} E B_{1}+B_{3}^{T} F B_{1}+B_{3}^{T} H B_{3} \\
+B_{0 \gamma}^{T} \bar{A} B_{0 \gamma}+B_{0 \gamma}^{T} \bar{B} B_{2 \gamma}+B_{2 \gamma}^{T} \bar{B} B_{0 \gamma}+B_{2 \gamma}^{T} \bar{B} B_{2 \gamma}
\end{array}\right) \mathrm{d} S, \\
K_{u \phi e}^{a} & =\int_{S_{e}}\left(-B_{0}^{T} A e^{T} B_{\phi a}-B_{1}^{T} B e^{T} B_{\phi a}-B_{3}^{T} E e^{T} B_{\phi a}\right) \mathrm{d} S, \\
K_{u \phi e}^{s} & =\int_{S_{e}}\left(-B_{0}^{T} A e^{T} B_{\phi s}-B_{1}^{T} B e^{T} B_{\phi s}-B_{3}^{T} E e^{T} B_{\phi s}\right) \mathrm{d} S, \\
K_{\phi u e}^{a} & =\int_{S_{e}}\left(-B_{\phi a}^{T} e A B_{0}-B_{\phi a}^{T} e B B_{1}-B_{\phi a}^{T} e E B_{3}\right) \mathrm{d} S, \\
K_{\phi u e}^{s} & =\int_{S_{e}}\left(-B_{\phi s}^{T} e A B_{0}-B_{\phi s}^{T} e B B_{1}-B_{\phi s}^{T} e E B_{3}\right) \mathrm{d} S, \\
K_{\phi \phi}^{a} e & =\int_{S_{e}} B_{\phi a}^{T} p B_{\phi a}^{T} \mathrm{~d} S, \\
K_{\phi \phi}^{s} e & =\int_{S_{e}} B_{\phi s}^{T} p B_{\phi s}^{T} \mathrm{~d} S, \\
P_{e} & =\int_{S_{e}} N^{T} \widehat{F} \cdot \mathrm{d} S, \\
F_{q e}^{a} & =\int_{S_{e}} B_{\phi}^{a T} Q^{a} \mathrm{~d} S, \\
F_{q e}^{s} & =\int_{S_{e}} B_{\phi}^{s T} Q^{s} \mathrm{~d} S,
\end{aligned}
$$

where $Q^{b}$ and $Q^{a}$ are the electric potentials, which act on the sensor and actuator layers, respectively.

The expression of the kinetic energy of the plate element is expressed as

$$
\begin{aligned}
T_{e} & =\frac{1}{2} \int_{V_{e}} \dot{u}^{T} \rho(x, z) \dot{u} \mathrm{~d} V \\
& =\frac{1}{2} \dot{q}_{e}^{T}\left(\begin{array}{l}
\int_{V_{e}} N^{T} L^{T} \rho(x, y) L N \mathrm{~d} V+\int_{V_{e}} N^{T} L^{T} \rho_{a} L N \mathrm{~d} V \\
\int_{V_{e}} N^{T} L^{T} \rho_{s} L N \mathrm{~d} V
\end{array}\right) \dot{q}_{e} \\
& =\frac{1}{2} \dot{q}_{e}^{T} M_{e} \dot{q}_{e},
\end{aligned}
$$

where $\rho, \rho_{b}$, and $\rho_{a}$ are, respectively, the densities of the core, sensor, and actuator layers, $N$ is Lagrangian shape function matrices, and $L$ and $M_{\mathrm{e}}$ are calculated as

$$
L=\left[\begin{array}{ccccc}
1 & 0 & -\frac{4 z^{3}}{3 h^{2}} \frac{\partial}{\partial x} & z-\frac{4 z^{3}}{3 h^{2}} & 0 \\
0 & 1 & -\frac{4 z^{3}}{3 h^{2}} \frac{\partial}{\partial y} & 0 & z-\frac{4 z^{3}}{3 h^{2}} \\
0 & 0 & 1 & 0 & 0
\end{array}\right],
$$

$$
M_{e}=\left(\begin{array}{l}
\int_{V_{e}} N^{T} L^{T} \rho(x, y) L N \mathrm{~d} V+\int_{V_{e}} N^{T} L^{T} \rho_{a} L N \mathrm{~d} V \\
\int_{V_{e}} N^{T} L^{T} \rho_{s} L N \mathrm{~d} V
\end{array}\right) .
$$

Then, the weak form type Galerkin of the controlling equations of the plate can be formed by employing Hamilton's principle, where it can be expressed as

$$
\delta L_{e}=0 \text {, }
$$

in which

$$
L_{e}=T_{e}-\prod_{e}
$$

Substituting the expressions of the kinetic function into equation (28), then group them individually into the displacement and potential variables, and the resulting equation is expressed as

$$
\left[\begin{array}{ccc}
M_{u e} & 0 & 0 \\
0 & 0 & 0 \\
0 & 0 & 0
\end{array}\right]\left\{\ddot{q}_{e} \ddot{\phi}_{a e} \ddot{\phi}_{s e}\right\}+\left[\begin{array}{ccc}
K_{u u e} & K_{u \phi e}^{a} & K_{u \phi e}^{s} \\
K_{\phi u e}^{a} & K_{\phi \phi e}^{a} & 0 \\
K_{\phi u e}^{s} & 0 & K_{\phi \phi e}^{s}
\end{array}\right]\left\{\begin{array}{c}
q_{e} \\
\phi_{a e} \\
\phi_{s e}
\end{array}\right\}=\left\{\begin{array}{c}
P_{e} \\
F_{q e}^{a} \\
F_{q e}^{s}
\end{array}\right\} .
$$

From the second and third equations in (29), $\phi_{a e}$ and $\phi_{s e}$ can be obtained; then, adding them into the first expression, the forced oscillation equation of the plate element in the matrix form is obtained as

$$
M_{u e} \ddot{q}_{e}+\left(\begin{array}{c}
K_{u u e}+K_{u \phi e}^{a} K_{\phi \phi e}^{a-1} K_{\phi u e}^{a} \\
+K_{u \phi e}^{s} K_{\phi \phi-e}^{s-1} K_{\phi u e}^{s}
\end{array}\right) q_{e}=P_{e}+K_{u \phi e}^{s} F_{q e}^{s}+K_{u \phi e}^{a} F_{q e}^{a} .
$$

When the external load acts on the structure, the structure is going to be bent; thus, the voltage will be varied; then, the voltage of the sensor will be passed to the controlling center to restrain the acting voltage return to the actuator one to derive an electric load that proceeds opposing to the way, where the structure is bending, getting back the structure tlo its balance before the deformation phenomenon. The operation is expressed as presented in Figure 2.

Now, consider $G_{v}$ as a velocity feedback coefficient and $G_{d}$ as a displacement reaction coefficient of the controlling station, and these reaction coefficients deputize the relation in the middle of the input voltage $\phi_{a}$ and the output voltage in the piezoelectric sensor layer $\phi_{s}$ :

$$
\phi_{a e}=G_{d} \phi_{s e}+G_{v} \dot{\phi}_{s e}
$$

Herein, by neglecting the aspect of the concerned $Q^{a}$ and $Q^{s}$, from the third-expression of equation (29), the voltage vector of the piezoelectric sensor component is calculated as follows: 


$$
\phi_{s e}=K_{\phi \phi}^{s-1} K_{u \phi}^{s} q_{e} .
$$

And, the induced charge due to the deformation is

$$
Q_{e}^{s}=K_{u \phi e}^{s} q_{e} .
$$

This can be explained that, as the system is vibrated, the voltage engendered in the piezoelectric sensor component due to the strain phenomenon of the structure is about to engender an initial indication to the controlling station, which is going to affect directly on the piezoelectric actuator layer. Therefore, the stress and strain components due to the piezoelectricity will generate a voltage indication applied back to it, which controls the strain and stress in the desired way.

By adding equations (31) and (32) into the second and third equations in (29), we obtain the following expression:

$$
Q_{e}^{a}=K_{u \phi e}^{a} q_{e}-G_{d} K_{u \phi}^{a} K_{\phi \phi}^{s} e_{-}^{1} K_{\phi u e}^{s} q_{e}-G_{v} K_{u \phi e}^{a} K_{\phi \phi}^{s} e_{-}^{1} K_{\phi u e}^{s} \dot{q}_{e} .
$$

Finally, by adding equation (34) into equation (30), the forced oscillation equation of plate element is obtained as

$$
M_{u e} \ddot{q}_{e}+C_{e}^{d} \dot{q}_{e}+K_{e}^{*} q_{e}=P_{e}
$$

in which

$$
K_{e}^{*}=K_{u u e}+G_{d} K_{u \phi e}^{a} K_{\phi \phi}^{s} e_{-}^{1} K_{\phi u e}^{s} .
$$

and $C_{e}^{d}$ is the damping matrix caused by the influence of the feedback parameter, which is defined as

$$
C_{e}^{d}=G_{v} K_{u \phi e}^{a} K_{\phi \phi}^{s} e_{-}^{1} K_{\phi u e}^{s} .
$$

Neglecting the damping coefficient generated by the structure, the forced oscillation equation of the element is expressed as

$$
M_{u e} \ddot{q}_{e}+\left(C_{e}^{d}+C_{e}\right) \dot{q}_{e}+K_{e}^{*} q_{e}=P_{e}
$$

where $C_{e}$ is the Rayleigh structural dampness matrix, where it is computed by the mass and stiffness matrices:

$$
C_{e}=\bar{\alpha} M_{u u e}+\bar{\beta} K_{u u e} .
$$

By operating the process of coming together with the element vectors and matrices and deleting the fixed DOFs, the forced oscillation equation of the piezoelectric structure is obtained as follows:

$$
M_{u} \ddot{q}+\left(C^{d}+C\right) \dot{q}+K^{*} q=\breve{P} .
$$

\section{Numerical Results and Discussion}

\subsection{Static of a Piezoelectric 2D-FGM Plate}

4.1.1. Accuracy Study. Firstly, the computed results of the present approach are collated with those of the CS-DSG3 [20]. A piezoelectric $\mathrm{FG}$ plate $\left(\mathrm{Ti}-6 \mathrm{Al}-4 \mathrm{~V} / \mathrm{Al}_{2} \mathrm{O}_{3}\right)$ with $a=b=0.2 \mathrm{~m}$, the depth $h_{p}=1 \mathrm{~mm}$, and substance

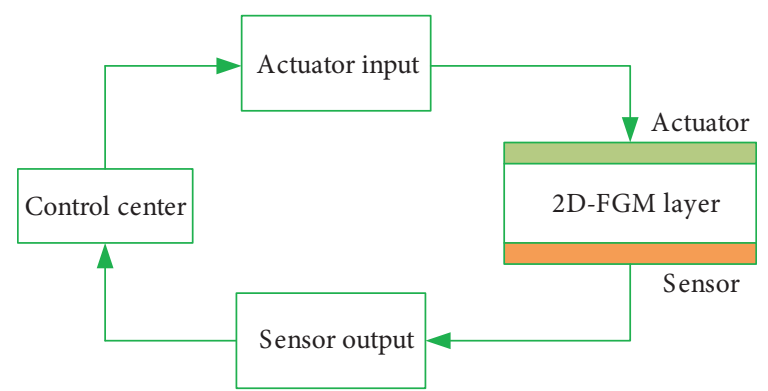

Figure 2: The oscillation control station of the piezoelectric plate.

characteristics $E_{c}=320.24 \mathrm{GPa}, v_{c}=0.26, \rho_{c}=3750 \mathrm{~kg} / \mathrm{m}^{3}$, $E_{m}=105.7 \mathrm{GPa}, \nu_{m}=0.2981$, and $\rho_{m}=4429 \mathrm{~kg} / \mathrm{m}^{3}$ is considered. The thicknesses of two piezoelectric layers at the top and bottom surfaces are $h_{a}=h_{s}=0.1 \mathrm{~mm}$; the piezoelectric mechanical characteristics are $E_{p i}=63 \mathrm{GPa}, v_{p i}=0.3$, $d_{31}=d_{32}=254.10^{-12} \mathrm{~m} / \mathrm{V}$, and $k_{33}=15 \mathrm{nF} / \mathrm{m}$. The structure is thoroughly simply constrained (SSSS). As the volume fraction exponent $n=2, G_{d}=5$, and the deflection of the plate at $y=b / 2$ is presented in Figure 3 with different mesh sizes, and compared with those of Nguyen-Quang et al. [20], one can see that the mesh size is increased (the number of elements increases) and the deflection curve of the plate converges to the result shown in document [20]. It can be seen that, with the mesh size $8 \times 8$, the numerical data of this work meets a good agreement. Therefore, for all following explorations, this mesh size will be employed.

4.1.2. Computed Results of Static Bending of Piezoelectric 2DFGM Plates. Now, a square plate structure $(a=b=0.2 \mathrm{~m}$ and thickness $h_{p}=1 \mathrm{~mm}$ ) is considered The actuator and sensor layers have the same thickness $h_{a}=h_{s}=0.1 \mathrm{~mm}$. The uniformly distributed load is $q_{0}$, and the substance characteristics of the structure are $E_{1}=205.1 \mathrm{GPa}, E_{2}=70 \mathrm{GPa}, E_{3}=151 \mathrm{GPa}, v_{1}=$ $0.3, v_{2}=0.3$, and $v_{3}=0.3$. The piezoelectric layers are defined as $E_{p i}=63 \mathrm{GPa}, v_{p i}=0.3, \rho_{a}=\rho_{s}=7600 \mathrm{~kg} / \mathrm{m}^{3}, k_{33}=15 \mathrm{nF} / \mathrm{m}$, and $d_{31}=d_{32}=254.10^{-12} \mathrm{~m} / \mathrm{V}$. Two boundary conditions are used: fully simply supported (SSSS) and one clamped edge and three free edges (CFFF). To be more convenient, the nondimensional deflection is normalized as follows:

$$
w^{*}=\frac{10^{3} D}{q_{0} a^{4}} w_{\min },
$$

with

$$
\mathrm{D}=\frac{E_{1} h^{3}}{12\left(1-v_{1}^{2}\right)}
$$

(1) Effect of the Volume Fraction Indices $n$ and $q$. By changing the values of volume fraction indices $n$ and $q$ in the range of 0 to 10 , the nondimensional deflection $w^{*}$ is plotted in Figure 4 . When $n$ varies from 0 to 2 , if the value of $q$ increases, the nondimensional deflection $w^{*}$ will increase. However, when the value of $n$ is higher than 2, the increase of $q$ will guide to a decrease in the nondimensional deflection $w^{*}$. This is because changing both $n$ 


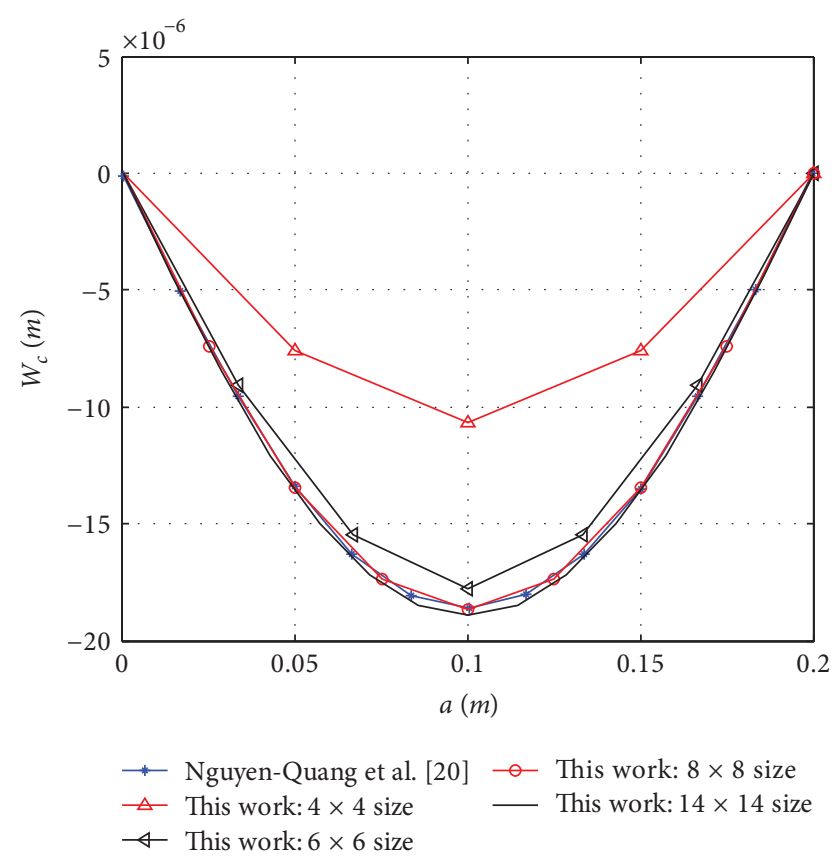

FIGURE 3: Comparison deflection of the piezoelectric 2D-FGM plate, $G_{d}=5$.

and $q$ results in a change in the substance characteristics; therefore, the mechanical behaviors of the structure are also changed.

(2) Effect of the Feedback Coefficient $G_{d}$. Figure 5 presents the nondimensional defection of the plate at $y=b / 2$ with the value of the feedback coefficient $G_{d}$ varies in a range of 0 to 10 for the SSSS plate $(n=10$ and $q=0.5)$. When increasing $G_{d}$, the maximum deflection of the plate decreases, and the deflection line is no longer symmetrical through the $x=a / 2$. This is due to the fact that the material also varies in the $O x$ direction of the plate. Figures 5-8 present the deflection of the plate at $y=b / 2$ for the CFFF plate. As the reaction coefficient $G_{d}$ increases, the nondimensional maximum deviation of the structure degrades. This can be illuminated that, due to the increase of $G_{d}$, the stiffness matrix is increased by an amount as presented in equation (36), so the structure becomes stiffer and causes the deflection to decrease.

\subsection{Numerical Results and Discussion on the Dynamic Response of Piezoelectric FG Plates}

4.2.1. Accuracy Study. This section carries out a comparison of the dynamic response of the CFFF square piezoelectric FG plate (Ti-6Al-4V/Al $\mathrm{O}_{2}$ ) with $a=b=0.2 \mathrm{~m}$ and the plate depth $h=20 \mathrm{~mm}$; two piezoelectric layers have the same thickness $h_{a}=h_{s}=2 \mathrm{~mm}$. The characteristics of the piezoelectric layers are presented in this section.

The load is changed over time by the following formula:

$$
P=P_{0} \sin \left(\frac{\pi x}{a}\right) \sin \left(\frac{\pi y}{b}\right) F(t),
$$

with the load amplitude $P_{0}=10^{4} \mathrm{~N} / \mathrm{m}^{2}$, and the function $F(t)$ is expressed as

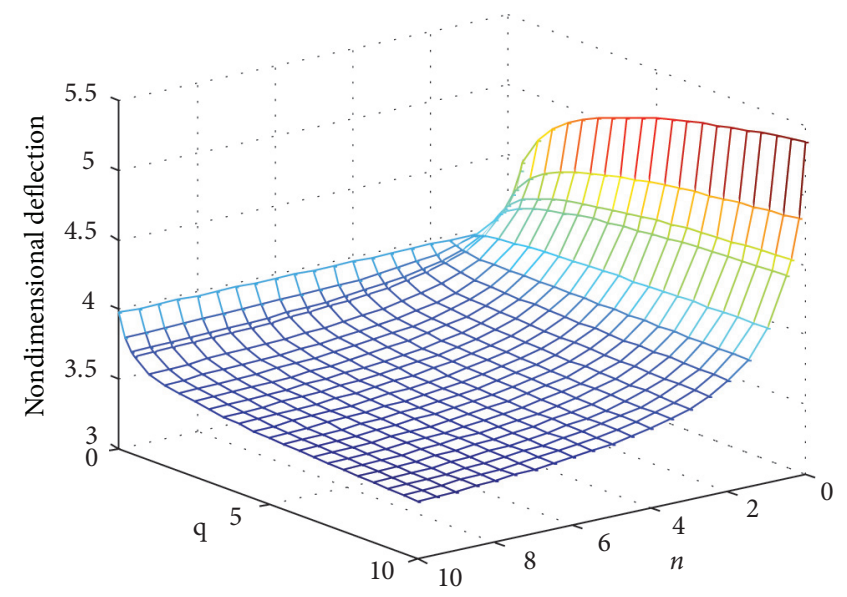

FIGURE 4: Nondimensional deflection $w^{*}$ depends on $n$ and $q$ SSSS, $G_{d}=0.5$ and $V=0$.

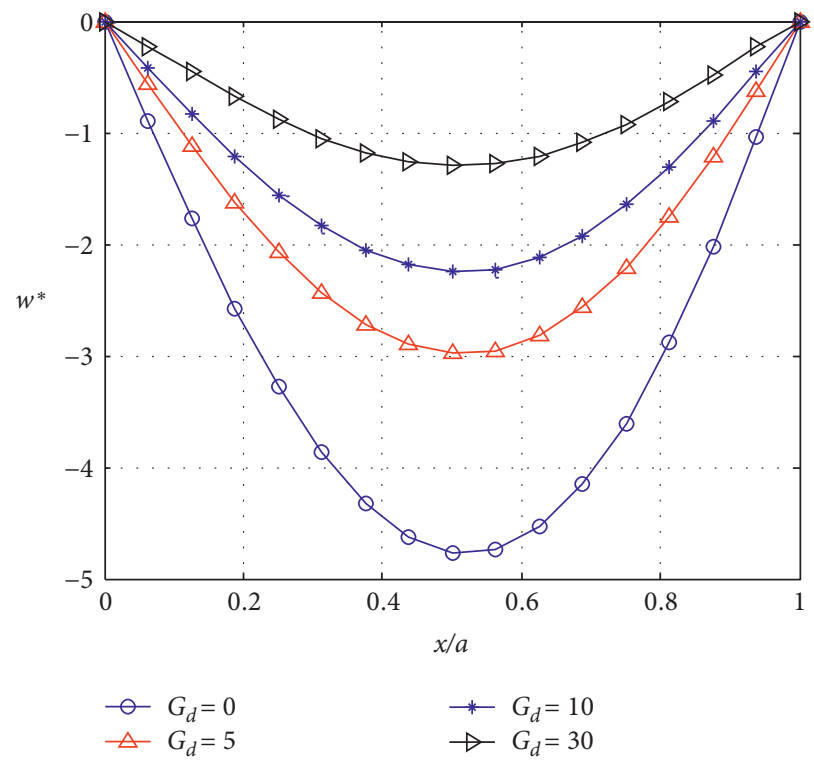

FIGURE 5: Nondimensional deflection $w^{*}$ of the SSSS plate depends on $G_{d}, n=10, q=0.5$, and $V=0$.

$$
F(t)= \begin{cases}\sin \left(\pi \frac{t}{t_{1}}\right), & 0 \leq t \leq t_{1}, \\ 0, & t>t_{1},\end{cases}
$$

where $t_{1}=2 \mathrm{~ms}$.

The comparative results of dynamic behaviors of the center point of the piezoelectric FG plate are shown in Figure 9 corresponding to the case of $G_{v}=0$ and $G_{d}=0$ and compared with those of the CS-DSG3 [20]. Readers can see that the present theory and mechanical model are verified.

4.2.2. Numerical Results and Discussion on the Dynamic Response of Piezoelectric 2D-FG Plates. In this section, the 


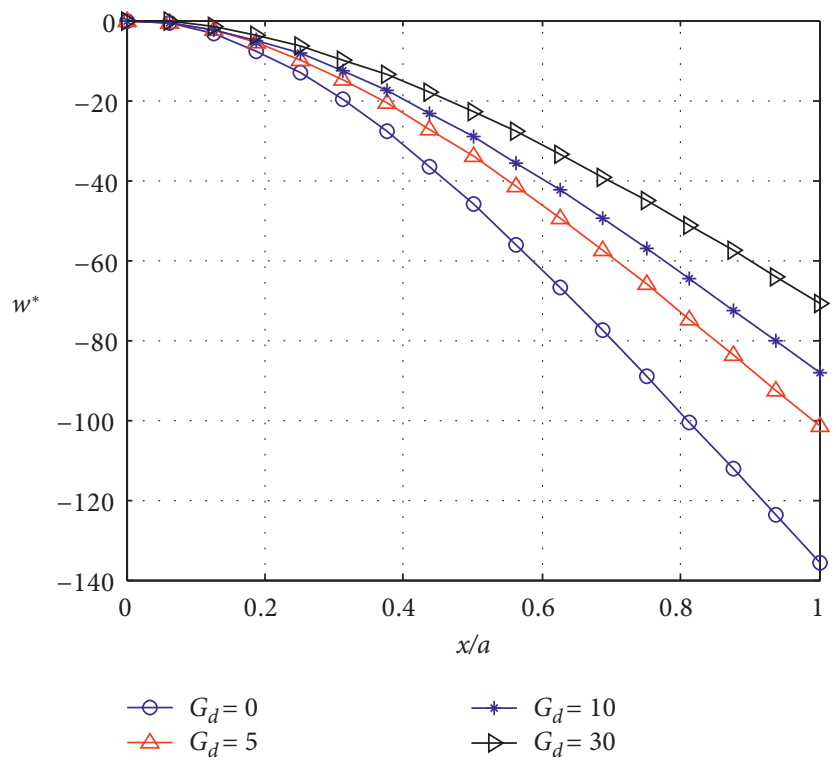

Figure 6: Nondimensional deflection $w^{*}$ of the CFFF plate depends on $G_{d}, n=10, q=0.5$, and $V=0$.

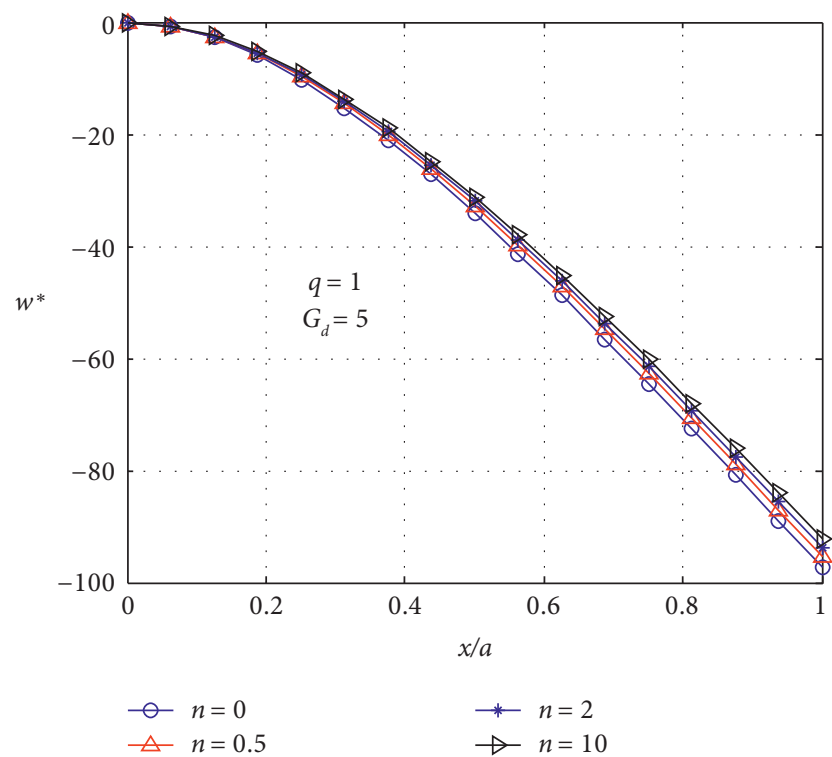

Figure 7: Nondimensional deviation $w^{*}$ of the CFFF plate depends on volume proportion index $n, G_{d}=5, q=1$, and $V=0$.

geometrical and material study is carried out. The reactions of geometric and mechanical parameters on the dynamic behaviors of the piezoelectric $2 \mathrm{D}$-FGM plate are investigated. The dimensions of the plate are $a=b=0.2 \mathrm{~m}$ and the plate depth $h=20 \mathrm{~mm}$. The mechanical characteristics of FG material are $E_{1}=205.1 \mathrm{GPa}, E_{2}=70 \mathrm{GPa}, E_{3}=151 \mathrm{GPa}, \nu_{1}=0.3, v_{2}=0.3$, and $\nu_{3}=0.3$. The outer surfaces are G-1195N (piezoelectric material) with the depths $h_{a}=h_{s}=2 \mathrm{~mm}$ and piezoelectric characteristics are $\quad E_{p i}=63 \mathrm{GPa}, \quad v_{p i}=0.3$, $d_{31}=d_{32}=254.10^{-12} \mathrm{~m} / \mathrm{V}, \rho_{p i}=7600 \mathrm{~kg} / \mathrm{m}^{3}$, and $k_{33}=15 \mathrm{nF} /$ $\mathrm{m}$. The sinusoidally distributed load is given as shown in equation (43). The CFFF boundary condition is used;

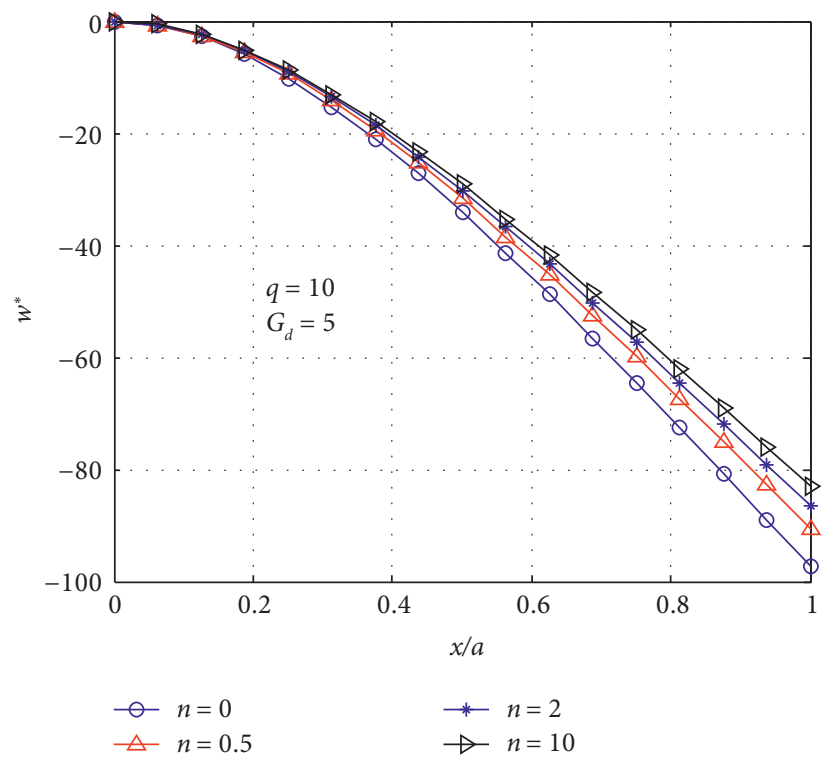

FIgURE 8: Nondimensional deviation $w^{*}$ of the CFFF plate depends on volume proportion index $n, G_{d}=5, q=10$, and $V=0$.

nondimensional deflection $w^{*}$ is defined in equation (41). In this problem, the Newmark- $\beta$ method is employed to solve the dynamic equation with the parameters $\bar{\beta}=0.25$ and $\bar{\alpha}=0.5$.

(1) Effect of the Feedback Coefficient $G_{v}$. Now, to examine the influence of $G_{V}$ on the proficiency and to eliminate the vibration of the structure, the plate with $n=2$ and $q=1$ is considered. Nondimensional deflexion $w^{*}$ of the center location of the outermost edge of the plate $(x=a, y=b / 2)$ is presented in Figures 10 and 11. The results indicate that

As the value of the response coefficient $G_{v}$ increases, nondimensional deflection $w^{*}$ will progress to 0 faster. This 


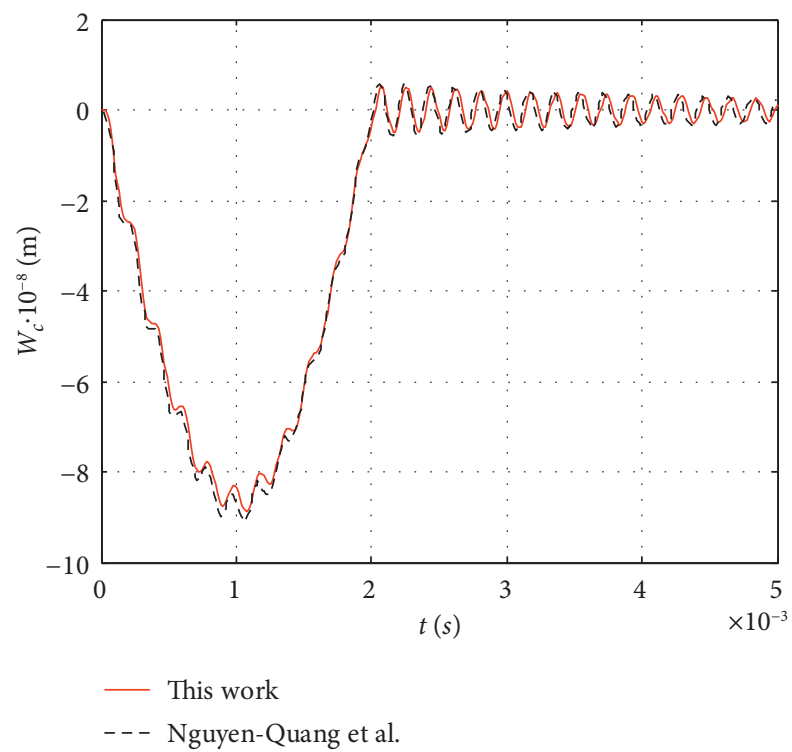

FIGURE 9: Nondimensional deflection of the center point of the piezoelectric FG under the sinusoidal load, $G_{v}=0, G_{d}=0$, and $n=2$.

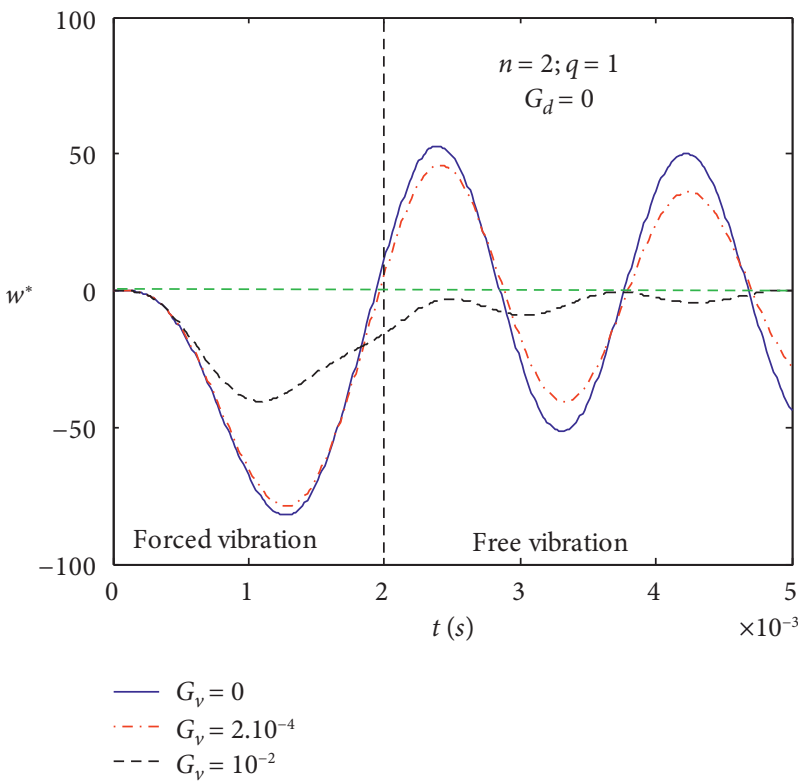

FIgURE 10: The dependence of the nondimensional deflection $w^{*}$ on $G_{v}, G_{d}=0, n=2$, and $q=1$, CFFF.

means that the proficiency to eliminate the vibration of the plate is better. At the same time, the maximum value of the nondimensional deflection $w^{*}$ also decreases, and this shows that the plate becomes stronger. This is because when $G_{v}$ is increased, the stiffness matrix of the structure is increased according to equation (37), causing the vibration energy of the plate to be dissipated; thus, the amplitude of the oscillation of the plate decreases.
As the value of the feedback coefficient $G_{V}$ becomes greater, the culmination value of nondimensional deflection $w^{*}$ decreases gradually.

(2) Influence of the Depth of the Piezoelectric Component. The changing the piezoelectric layer thickness $h_{s}$ will affect the overall stiffness and mass of the plate; therefore, it will significantly affect the response of the piezoelectric 2D-FGM 


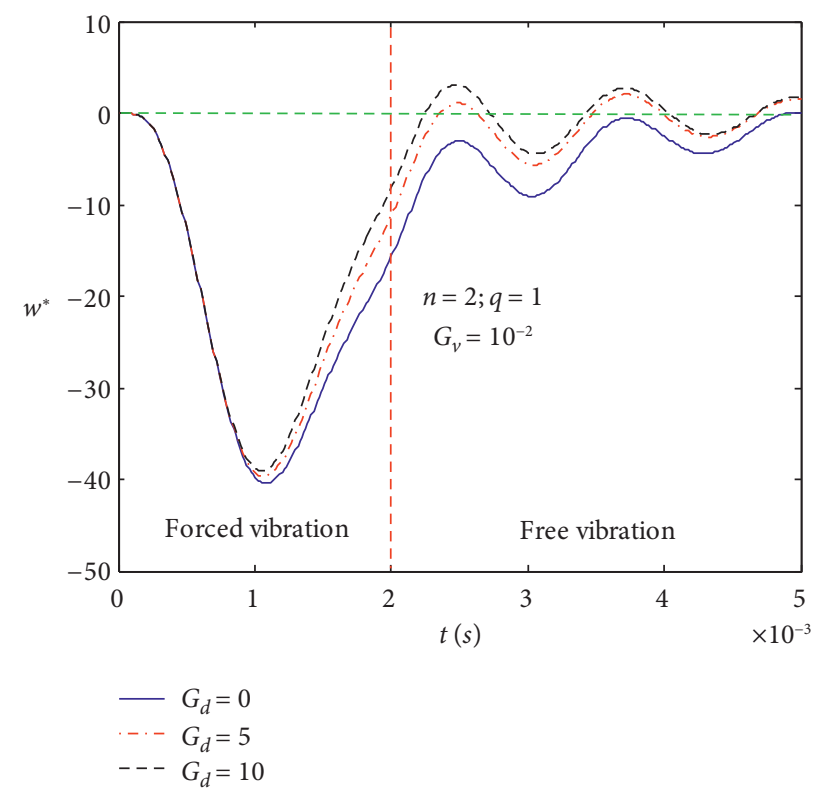

Figure 11: The dependence of the nondimensional deflection $w^{*}$ on the time $t, G_{d}, n=2$, and $q=1$, CFFF.

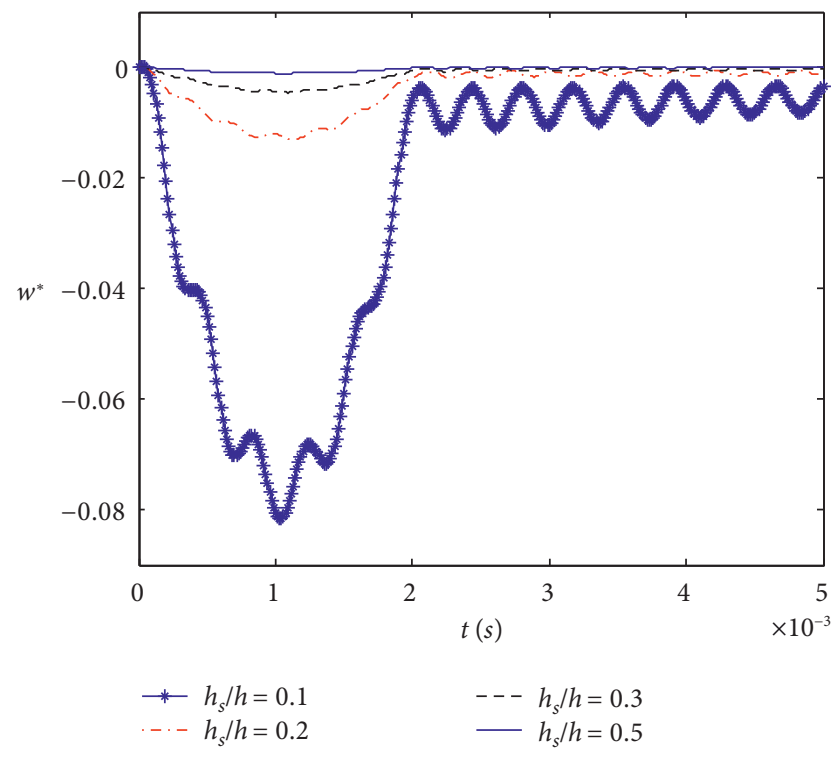

FIgURE 12: The changing of nondimensional deflection $w^{*}$ over time, $h_{s}=0.1-0.5, G_{\mathrm{d}}=0$ and $5, n=2, q=1$, and $G_{v}=10^{-2}$, CFFF.

plate. Figures 12-14 show the change of the nondimensional deflection $w^{*}$ over time as the depth of the piezoelectric layer $h_{s}$ is increased, $h_{s} / h=0.1-0.5$, respectively. Some discussions are given as follows:

(1) As the depth of the piezoelectric layer $h_{s}$ increases, maximum deflection $w^{*}$ decreases. At the same time, the shape of the deflection curve over time is also different. This is because both stiffness, mass, and structural damping matrices of the structure are

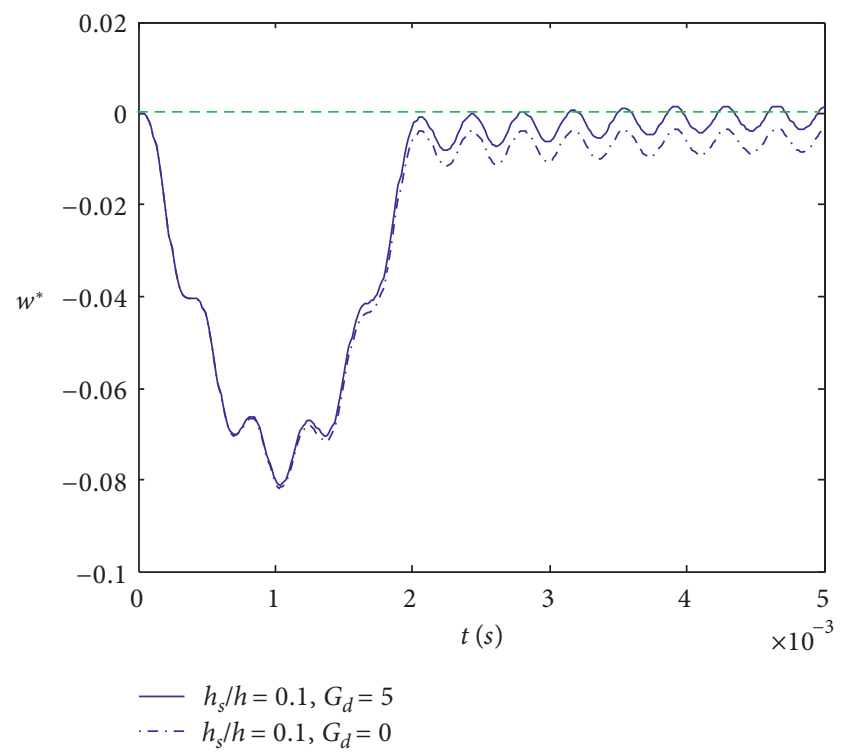

Figure 13: The changing of nondimensional deflection $w^{*}$ over time, $h_{s}=0.1, G_{\mathrm{d}}=0$ and $5, n=2, q=1$, and $G_{v}=10^{-2}$, CFFF.

changed, so both the maximum value and the responsive shape of the structure also change.

(2) When $h_{s} / h=0.1$ and 0.2 , changing the value of $G_{d}$ from $0-5$, it can be seen that the maximum value of the deflection $w^{*}$ does not change much, but when $G_{d}$ increases, nondimensional deflection $w^{*}$ will reach 0 faster. In addition, Figures $12-14$ show that, at the moment of the free oscillation, $w^{*}$ will 


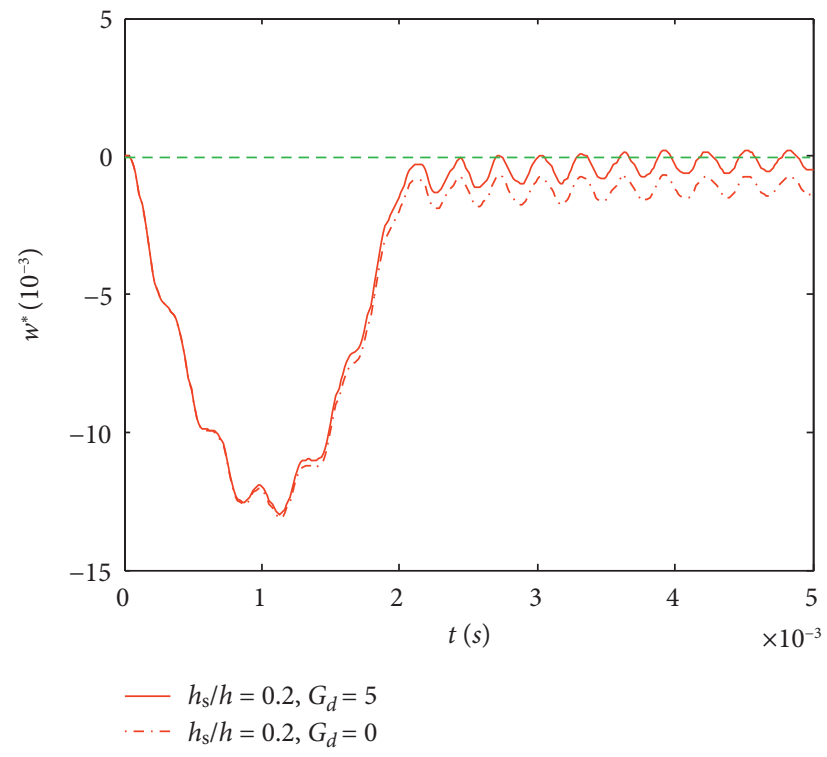

Figure 14: The changing of nondimensional deflection $w^{*}$ over time, $h_{s}=0.2, G_{\mathrm{d}}=0$ và $5, n=2, q=1$, and $G_{v}=10^{-2}$, CFFF.

approach 0 quickly or slowly depend on the value of $G_{d}$ and the piezoelectric layer thickness $h_{s}$.

\section{Conclusions}

This work carries out static bending and dynamic behavior analysis of piezoelectric 2D-FG plates by using the TSDT of Reddy and the FEM. The paper confirms the aptness of the present approach through comparison with the previously published data. Through the parameter study, some conclusions are highlighted as follows:

(1) As the value of the feedback coefficient $G_{d}$ becomes greater, the culmination deflexion of the structure decreases

(2) As the value of the feedback coefficient $G_{v}$ gets larger, the culmination deflexion of the structure decreases in the forced oscillation problem, and the vibration of the plate reduces faster, which is the advantage of the plate when the piezoelectric layer is integrated

(3) When the depth of the piezoelectricity component is boosted, the culmination deflection of the structure decreases, and the shape that responds to the deflection of the plate over time also changes.

\section{Data Availability}

The data used to support the findings of this study are included within the article.

\section{Conflicts of Interest}

The authors declare that they have no conflicts of interest.

\section{Acknowledgments}

Hoang Manh Hung gratefully acknowledges the support of Department for Standard Metrology and Quality, Foundation for Science and Technology Development, under Grant no. 2020/SMQ

\section{References}

[1] Y. Miyamoto, M. Niino, and M. Koizumi, "FGM research programs in Japan-from structural to functional uses," in Proceedings of the 4th International Symposium on Functionally Graded Materials, pp. 1-8, AIST Tsukuba Research Center, Tsukuba, Japan, October, 1996.

[2] H.-N. Nguyen, T. C. Tan, D. T. Luat, V.-D. Phan, D. V. Thom, and P. V. Minh, "Research on the buckling behavior of functionally graded plates with stiffeners based on the thirdorder shear deformation theory," Materials, vol. 12, no. 8, 1262 pages, 2019.

[3] N. Vu Hoai, D. Hong Doan, N. M. Khoa, T. Van Do, and H. Thi Tran, "Phase-field buckling analysis of cracked stiffened functionally graded plates," Composite Structures, vol. 217, pp. 50-59, 2019.

[4] L. K. Hoa, P. V. Vinh, N. D. Duc, N. T. Trung, L. T. Son, and D. V. Thom, "Bending and free vibration analyses of functionally graded material nanoplates via a novel nonlocal single variable shear deformation plate theory," Proceedings of the Institution of Mechanical Engineers, Part C: Journal of Mechanical Engineering Science, 2020.

[5] A. Saiyathibrahim, N. S. S. Mohamed, and Dhanapal, "Processing techniques of functionally graded materials-a review," in Proceedings of the International Conference on Systems, Science, Control, Communication, Engineering and Technology (ICSSCCET), Coimbatore, India, August 2015.

[6] N. A. Mahmoud, K. I. E. Ahmed, and I. Hassab-Allah, "Elastic-plastic analysis of two-dimensional functionally graded materials under thermal loading," International Journal of Solids and Structures, vol. 46, no. 14-15, pp. 2774-2786, 2009.

[7] N. Amir and M. H. Shojaeefard, "Elastic mechanical stress analysis in a 2D-FGM thick finite length hollow cylinder with newly developed material model," Acta Mechanica Solida Sinica, vol. 29, no. 2, pp. 178-191, 2016.

[8] H. S. Hedia, "Comparison of one-dimensional and two-dimensional functionally graded materials for the backing shell 
of the cemented acetabular cup," Journal of Biomedical Materials Research Part B: Applied Biomaterials, vol. 74B, no. 2, pp. 732-739, 2005.

[9] S. G. Pankaj, R. K. Vishesh, and P. E. Sudhagar, "On the numerical modelling and analysis of multi-directional functionally graded composite structures: a review," Composite Structures, vol. 236, Article ID 111837, 2020.

[10] V. D. Thom, K. N. Dinh, D. D. Nguyen, H. D. Duc, and Q. B. Tinh, "Analysis of bi-directional functionally graded plates by FEM and a new third-order shear deformation plate theory," Thin-Walled Structures, vol. 119, pp. 687-699, 2017.

[11] T. T. Tam, T. Nguyen-Thoi, and L. Jaehong, "Isogeometric size optimization of bi-directional functionally graded beams under static loads," Composite Structures, vol. 227, Article ID 111259, 2019.

[12] L. Jinqiang, X. Yu, L. Fengming, and N. Yoshihiro, "Active vibration control of functionally graded piezoelectric material plate," Composite Structures, vol. 207, pp. 509-518, 2019.

[13] R. L. Wankhade and K. M. Bajoria, "Vibration attenuation and dynamic control of piezolaminated plates with coupled electromechanical actuation," Archive of Applied Mechanics, vol. 91, pp. 411-426, 2020.

[14] V. N. Nam, L. Jaehong, and H. Nguyen-Xuan, "Active vibration control of GPLs-reinforced FG metal foam plates with piezoelectric sensor and actuator layers," Composites Part B: Engineering, vol. 172, pp. 769-784, 2019.

[15] M. H. Yas and N. Moloudi, "Three-dimensional free vibration analysis of multi-directional functionally graded piezoelectric annular plates on elastic foundations via state space based differential quadrature method," Applied Mathematics and Mechanics, vol. 36, no. 4, pp. 439-464, 2015.

[16] C. P. Wu, K. H. Chiu, and Y. M. Wang, "A review on the three-dimensional analytical approaches of multilayered and functionally graded piezoelectric plates and shells," Computers, Materials and Continua, vol. 8, no. 2, pp. 93-132, 2008.

[17] S. Yongbo, H. Shuguang, L. Yuping, X. Jibo, and L. Zhiyong, "The symplectic solution for the bi-directional functionally graded piezoelectric materials," Applied Mechanics and Materials, vol. 174-177, pp. 131-134, 2012.

[18] M. D. Rasool, R. Ali, and B. Kamran, "Damped dynamic behavior of an advanced piezoelectric sandwich plate," Composite Structures, vol. 243, Article ID 112243, 2020.

[19] X. D. Wang and G. L. Huang, "On the dynamic behavior of piezoelectric sensors and actuators embedded in elastic media," Acta Mechanica, vol. 197, no. 1-2, pp. 1-17, 2008.

[20] K. Nguyen-Quang, H. Dang-Trung, V. Ho-Huu, H. LuongVan, and T. Nguyen-Thoi, "Analysis and control of FGM plates integrated with piezoelectric sensors and actuators using cell-based smoothed discrete shear gap method (CSDSG3)," Composite Structures, vol. 165, pp. 115-129, 2017.

[21] M. Y. Yasin, N. Ahmad, and M. N. Alam, "Finite element analysis of actively controlled smart plate with patched actuators and sensors," Latin American Journal of Solids and Structures, vol. 7, no. 3, pp. 227-247, 2010.

[22] S. Valliappan and K. Qi, "Finite element analysis of a 'smart' damper for seismic structural control," Computers \& Structures, vol. 81, no. 8-11, pp. 1009-1017, 2003.

[23] H. S. Tzou and C. I. Tseng, "Distributed piezoelectric sensor/ actuator design for dynamic measurement/control of distributed parameter systems: a piezoelectric finite element approach," Journal of Sound and Vibration, vol. 138, no. 1, pp. 17-34, 1990.
[24] J. N. Reddy, “Analysis of functionally graded plates," International Journal for Numerical Methods in Engineering, vol. 47, no. 1-3, pp. 663-684, 2000.

[25] M. Arefi and I. Nahas, "Nonlinear electro thermo elastic analysis of a thick spherical functionally graded piezoelectric shell," Composite Structures, vol. 118, pp. 510-518, 2014.

[26] M. Arefi and G. H. Rahimi, "Non linear analysis of a functionally graded square plate with two smart layers as sensor and actuator under normal pressure," Smart Structures and Systems, vol. 8, no. 5, pp. 433-447, 2011.

[27] G. Moustafa, S. Hayat, and B. Fouad, "Influences of porosity distributions and boundary conditions on mechanical bending response of functionally graded plates resting on pasternak foundation," Steel and Composite Structures, vol. 38, no. 1, pp. 1-15, 2021.

[28] H. L. B. Tahar, F. Bouazza, and A. B. Abdelmoumen, "Porosity-dependent mechanical behaviors of FG plate using refined trigonometric shear deformation theory," Computers and Concrete, vol. 26, no. 5, pp. 439-450, 2020.

[29] C. C. Sara, K. Abdelhakim, and A. B. Abdelmoumen, "A novel four-unknown integral model for buckling response of FG sandwich plates resting on elastic foundations under various boundary conditions using Galerkinl's approach," Geomechanics and Engineering, vol. 21, no. 5, pp. 471-487, 2020.

[30] C. C. Sara, K. Abdelhakim, A. B. Abdelmoumen et al., "A novel four-unknown integral model for buckling response of FG sandwich plates resting on elastic foundations under various boundary conditions using Galerkinl's approach," Geomechanics and Engineering, vol. 21, no. 5, pp. 471-487, 2020. 